\title{
Phenomenological foundation and mathematical theory of sedimentation-consolidation processes
}

\author{
R. Bürger* \\ Institute of Mathematics A, University of Stuttgart, Pfaffenwaldring 57, D-70569 Stuttgart, Germany
}

\begin{abstract}
The phenomenological theory of sedimentation describes a flocculated suspension as a mixture of the solid and the fluid as two superimposed continuous media. Starting from the mass and linear momentum balances for each component, this theory yields, through constitutive assumptions and an order-of-magnitude analysis, three coupled partial differential equations describing the sedimentation-consolidation behaviour of the suspension in several space dimensions. The study and numerical solution of this system of equations has started only recently, but results are available for the one-dimensional case, in which these modelling equations reduce to a scalar hyperbolic-parabolic strongly degenerate partial differential equation with appropriate initial and boundary conditions. In this contribution, the research work performed by several groups of mathematicians on the formulation, analysis and numerical solution of mathematical sedimentation models is reviewed, with an emphasis on theoretical and numerical results for the simulation of the behaviour of compressible slurries. (C) 2000 Elsevier Science B.V. All rights reserved.
\end{abstract}

Keywords: Phenomenological theory; Sedimentation-consolidation process; Flocculated suspension; Theory of mixtures; Model equations

\section{Introduction}

Mathematical models for the simulation, the design and the control of continuous thickeners (see Fig. 1) are of great theoretical, practical and economical interest. During the last 20 years, the phenomenological theory of sedimentation has evolved as a widely accepted mathematical framework for the description of sedimentation-consolidation processes of flocculated suspensions. This theory is based on the theory of mixtures and describes a solid-liquid suspension as a mixture of two superimposed continuous media. The starting point of the modelling are the mass and linear momentum balances of both components. The application of constitutive assumptions, an order-of-magnitude study and the restriction of the motion to one space dimension reduce this set of equations to one scalar partial differential equation for the volumetric solids concentration plus an algebraic relationship for the excess pore pressure.

Clearly, the kinematic sedimentation model by Kynch [1] and its extensions to continuous thickening can now be viewed as a special case of the phenomenological model; Kynch's simple model is obtained if only the local mass balances are considered. Its major shortcoming is that it predicts that concentration values always propagate along

\footnotetext{
* Tel.: +49-711-6857647; fax: +49-711-6855599.

E-mail address: buerger@mathematik.uni-stuttgart.de (R. Bürger).
}

straight-lined characteristics, which might interact and thus produce discontinuities. It has long been known, however, that most real and, in particular, flocculated slurries such as the materials used in the mineral industries show different behaviour [2], they form compressible sediment layers characterised by curved iso-concentration lines, which Kynch's model is unable to predict. The development of the phenomenological theory has been very much inspirited by the desire to have a theory explaining this behaviour of real slurries in a satisfactory way, but which would at the same time yield a mathematical model that could be solved with reasonable effort and therefore be implemented as a simulation tool.

The phenomenological theory has in some variants been proposed by several authors, for example by Auzerais et al. [3], Buscall and White [4], Bascur [5], Concha and Bustos [6] and Landman and White [7] and has become widely accepted. However, mathematical analysis of the sedimentation-consolidation model emerging from this theory had been lacking for a long time, and experimental information had only rarely been used to validate this theory.

It was Bustos who started in her doctoral thesis [8], presented in 1984, applying mathematical results of the theory of hyperbolic conservation laws, which were very recent then, to Kynch's sedimentation model. Her work, guided by Wendland who later also became the author's advisor (see [9]) laid the foundation of an intense research 


\begin{tabular}{|c|c|}
\hline \multicolumn{2}{|c|}{ Nomenclature } \\
\hline \multicolumn{2}{|c|}{ Latin symbols } \\
\hline$a(\phi)$ & diffusion coefficient \\
\hline$A(\phi)$ & primitive of $a(\phi)$ \\
\hline b & external body force \\
\hline$C$ & $\begin{array}{l}\text { parameter in Michaels and Bolger's } \\
\text { approach for } f_{\mathrm{bk}}\end{array}$ \\
\hline$d$ & characteristic size of a single floc \\
\hline$f_{\mathrm{bk}}(\phi)$ & Kynch batch flux density function \\
\hline$f_{\mathrm{bk}}^{*}(\phi)$ & $\begin{array}{l}\text { dimensionless Kynch batch } \\
\text { flux density function }\end{array}$ \\
\hline$f_{\mathrm{F}}(t)$ & feed flux \\
\hline $\mathrm{Fr}$ & Froude number of the flow \\
\hline$g$ & acceleration due to gravity \\
\hline$h(\phi)$ & ratio $\mu_{\mathrm{s}}(\phi) / \mu_{\operatorname{mix}}(\phi)$ \\
\hline I & identity tensor \\
\hline$k$ & $\begin{array}{l}\text { parameter in semi-empirical approach } \\
\text { for } \sigma_{\mathrm{e}}\end{array}$ \\
\hline $\mathbf{k}$ & upwards pointing unit vector \\
\hline$L$ & depth of the sedimentation vessel \\
\hline $\mathbf{m}$ & $\begin{array}{l}\text { solid-fluid interaction force } \\
\text { per unit volume }\end{array}$ \\
\hline $\mathbf{m}_{\mathrm{b}}$ & hydrostatic part of $\mathbf{m}$ \\
\hline $\mathbf{m}_{\mathrm{d}}$ & dynamic part of $\mathbf{m}$ \\
\hline$n$ & $\begin{array}{l}\text { parameter in semi-empirical } \\
\text { approach for } \sigma_{\mathrm{e}}\end{array}$ \\
\hline $\mathbf{n}_{\mathbf{I}}$ & normal vector of singular surface \\
\hline$p$ & total pressure \\
\hline$p *$ & dimensionless total pressure \\
\hline$\tilde{p}$ & pore pressure \\
\hline$p_{\mathrm{e}}$ & excess pore pressure \\
\hline$p_{\mathrm{s}}, p_{\mathrm{s}}$ & solid/fluid component phase pressures \\
\hline$q, \mathbf{q}$ & volume average velocity \\
\hline$q^{*}$ & dimensionless volume average velocity \\
\hline $\mathbf{q}^{+}, \mathbf{q}^{-}$ & $\begin{array}{l}\text { approximate limits of } \mathbf{q} \text { at a singular } \\
\text { surface }\end{array}$ \\
\hline$Q_{\mathrm{D}}(t)$ & volumetric discharge rate \\
\hline $\bar{Q}_{T}$ & space-time cylinder \\
\hline$r(\phi)$ & auxiliary function \\
\hline $\operatorname{Re}_{\text {sed }}$ & sedimentation Reynolds number \\
\hline $\mathcal{S}$ & cross section of a settling column \\
\hline $\mathcal{S}_{\mathrm{f}}$ & part of $\mathcal{S}$ filled out by the fluid \\
\hline$t$ & time \\
\hline$t_{0}$ & characteristic time \\
\hline$T$ & maximum time \\
\hline $\mathbf{T}_{\mathrm{s}}, \mathbf{T}_{\mathrm{f}}$ & $\begin{array}{l}\text { solid/fluid component Cauchy } \\
\text { stress tensors }\end{array}$ \\
\hline $\mathbf{T}_{\mathrm{s}}^{\mathrm{E}}, \mathbf{T}_{\mathrm{f}}^{\mathrm{E}}$ & $\begin{array}{l}\text { solid/fluid component viscous } \\
\text { stress tensors }\end{array}$ \\
\hline $\mathbf{T}^{\mathrm{E}}$ & viscous stress tensor of the mixture \\
\hline $\mathbf{T}_{\mathrm{I}}^{\mathrm{E}}$ & inner part of $\mathbf{T}^{\mathrm{E}}$ \\
\hline$u^{*}$ & a dimensionless flow quantity \\
\hline$u_{\infty}$ & characteristic velocity of $\mathbf{v}_{\mathrm{r}}$ \\
\hline$U$ & characteristic velocity of $\mathbf{q}$ \\
\hline
\end{tabular}

\begin{tabular}{|c|c|}
\hline $\mathcal{U}$ & cross-sectional area of an ICT \\
\hline $\mathbf{v}$ & mass average velocity \\
\hline$v_{\infty}$ & $\begin{array}{l}\text { parameter in Michaels and Bolger's } \\
\text { approach for } f_{\mathrm{bk}}\end{array}$ \\
\hline$\nabla \mathbf{v}_{\operatorname{mix}}$ & $\begin{array}{l}\text { mixture representative velocity } \\
\text { gradient }\end{array}$ \\
\hline $\mathbf{v}_{\mathrm{s}}, \mathbf{v}_{\mathrm{f}}$ & solid/fluid phase velocities \\
\hline $\mathbf{v}_{\mathrm{r}}$ & drift velocity \\
\hline $\mathbf{v}_{\mathrm{r}}^{*}$ & dimensionless drift velocity \\
\hline$z$ & height \\
\hline \multicolumn{2}{|c|}{ Greek symbols } \\
\hline$\alpha_{1}, \alpha_{2}$ & multiindices (see Eq. (40)) \\
\hline$\alpha(\phi)$ & resistance coefficient \\
\hline$\beta(\phi)$ & coefficient in Eq. (9) \\
\hline$\gamma(\phi)$ & virtual mass (see Eq. (9)) \\
\hline$\gamma^{*}(\phi)$ & dimensionless virtual mass \\
\hline$\epsilon$ & surface porosity \\
\hline$\Delta \varrho$ & solid-fluid mass density difference \\
\hline$\phi$ & volumetric solids concentration \\
\hline$\phi_{0}(z)$ & initial concentration distribution \\
\hline$\phi_{\mathrm{c}}$ & critical concentration \\
\hline$\phi_{L}(t)$ & concentration prescribed at $z=L$ \\
\hline$\phi_{\max }$ & maximum concentration \\
\hline$\kappa_{\mathrm{s}}, \kappa_{\mathrm{f}}$ & $\begin{array}{l}\text { solid and fluid bulk or expansion } \\
\text { viscosities }\end{array}$ \\
\hline$\lambda_{\mathrm{s}}, \lambda_{\mathrm{f}}$ & functions related to phase viscosities \\
\hline$\mu_{0}$ & dynamic shear viscosity of the fluid \\
\hline$\mu_{\mathrm{s}}, \mu_{\mathrm{f}}$ & $\begin{array}{l}\text { solid/fluid component phase shear } \\
\text { viscosities }\end{array}$ \\
\hline$\mu_{\operatorname{mix}}(\phi)$ & mixture-representative shear viscosity \\
\hline & kinematic viscosity of the pure fluid \\
\hline $\boldsymbol{\Psi}_{1}, \boldsymbol{\Psi}_{2}$ & $\begin{array}{l}\text { non-linear functions defined in } \\
\text { Eqs. (41) and (42) }\end{array}$ \\
\hline$\varrho(\phi)$ & local density of the mixture \\
\hline$\varrho_{\mathrm{s}}, \varrho_{\mathrm{f}}$ & solid/fluid mass densities \\
\hline$\sigma$ & jump propagation velocity \\
\hline$\sigma_{\mathrm{e}}(\phi)$ & effective solid stress function \\
\hline$\sigma_{\mathrm{e}}^{*}(\phi)$ & $\begin{array}{l}\text { dimensionless effective solid } \\
\text { stress function }\end{array}$ \\
\hline
\end{tabular}

cooperation between the Universities of Concepción in Chile and Stuttgart in the topic of mathematical and numerical analysis of first the kinematic and then the phenomenological model of sedimentation. The joint research work has made it possible to put sedimentation under a solid and unified mathematical background. The results, originally published in numerous articles in different journals, have recently been cast into a monograph [10].

It is the purpose of this paper to present a brief outline first, in Section 2, of the phenomenological theory and then, in Section 3, of the basic mathematical concepts that have been employed for its analysis. It is pointed out that the model equations are derived here in several space dimensions, although the available analysis is still restricted to 


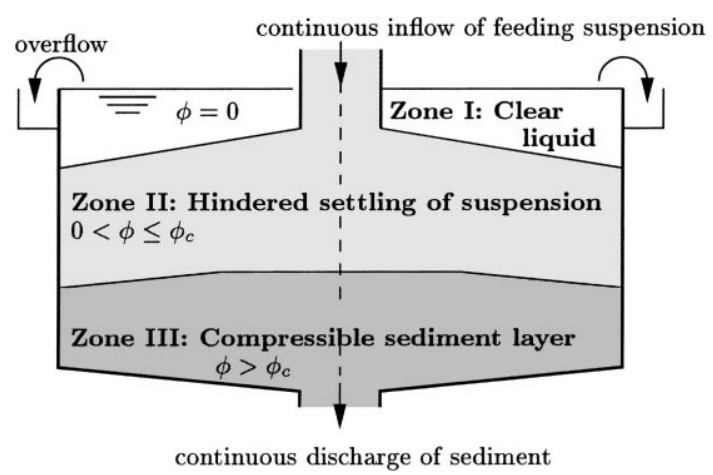

Fig. 1. Schematic view of a continuous thickener.

one space dimension. However, it seems that the extension to several space dimensions makes the modelling of new phenomena necessary, most notably the interaction of the concentration distribution with the average flow field, which does not occur in a single space dimension. Some of these issues, related to the current state of the phenomenological theory, are discussed in Section 4.

This paper is co-ordinated with the author's contributions with Evje et al. [11] and with Concha and Tiller [12] to this issue. In [11], attention is focused on the computational treatment of the field equation of the phenomenological model, which exhibits some unusual features making the application of simplistic standard schemes impossible. By comparison with published experimental information, we demonstrate in [12] that the phenomenological theory correctly predicts the sedimentation-consolidation behaviour of real slurries. Both companion contributions $[11,12]$ to this article provide calculated examples of batch and continuous sedimentation-consolidation processes. These examples are also meant to illustrate the material presented in this paper, for this reason and for sake of brevity, no computations are presented here.

\section{Phenomenological foundation of sedimentation-consolidation processes}

\subsection{Continuity equations and linear momentum balances of the components}

The decisive feature of the phenomenological theory is the description of all components as superimposed interacting continuous media according to the concepts of continuum mechanics $[13,14]$. The macroscopic equations are established as the fundamental equations, from which the local balances and jump conditions are derived. As has been argued elsewhere [15], the field variables used in this approach are equivalent to those obtained by starting from the equations of fluid mechanics on a particle scale, integrating them over some representative region and using the resulting space-averaged quantities as new field variables as proposed e.g. in $[16,17]$.
Here, we consider the sedimentation of a flocculated suspension of solid particles under the general assumptions that the solid particles are small with respect to the sedimentation vessel and have the same density; that the constituents of the suspension are incompressible; that the suspension is completely flocculated before the sedimentation begins; and that there is no mass transfer between the components during sedimentation.

\subsection{Solid and liquid component mass and linear momentum balances}

A mixture obeying such assumptions may be regarded as a superposition of two continuous media and can be described by the following field variables: the local volume fraction of solids $\phi$, the solid and fluid component velocities $\mathbf{v}_{\mathrm{s}}$ and $\mathbf{v}_{\mathrm{f}}$, the solid and fluid component Cauchy stress tensors $\mathbf{T}_{\mathrm{s}}$ and $\mathbf{T}_{\mathrm{f}}$, the external body force $\mathbf{b}$, which is assumed here to be gravity only, i.e. $\mathbf{b}=-g \mathbf{k}$ where $\mathbf{k}$ is the upwards-pointing unit vector, and the solid-fluid interaction force per unit volume $\mathbf{m}$. The macroscopic mass and linear momentum balances these variables obey are well known (see, e.g. [18-20]) and will not be written out here. The local balances in differential form are obtained by applying Gauss' theorem and the localisation theorem [21] to the macroscopic balances. Then, the continuity equations or local mass balances for the solid and liquid components are

$\frac{\partial \phi}{\partial t}+\nabla \cdot\left(\phi \mathbf{v}_{\mathrm{s}}\right)=0$

$\frac{\partial(1-\phi)}{\partial t}+\nabla \cdot\left((1-\phi) \mathbf{v}_{\mathrm{f}}\right)=0$

respectively. Using the volume-average flow velocity of the mixture, $\mathbf{q}:=\phi \mathbf{v}_{\mathrm{s}}+(1-\phi) \mathbf{v}_{\mathrm{f}}$, we obtain, by summing Eqs. (1) and (2), the volume continuity equation of the mixture,

$\nabla \cdot \mathbf{q}=0$.

In what follows, let Eq. (3) replace Eq. (2). The solid and liquid linear momentum balances can be written as

$$
\begin{aligned}
& \varrho_{\mathrm{s}} \phi\left(\frac{\partial \mathbf{v}_{\mathrm{s}}}{\partial t}+\left(\mathbf{v}_{\mathrm{s}} \cdot \nabla\right) \mathbf{v}_{\mathrm{s}}\right)=\nabla \cdot \mathbf{T}_{\mathrm{s}}+\varrho_{\mathrm{s}} \phi \mathbf{b}+\mathbf{m}, \\
& \varrho_{\mathrm{f}} \phi(1-\phi)\left(\frac{\partial \mathbf{v}_{\mathrm{f}}}{\partial t}+\left(\mathbf{v}_{\mathrm{f}} \cdot \nabla\right) \mathbf{v}_{\mathrm{f}}\right) \\
& \quad=\nabla \cdot \mathbf{T}_{\mathrm{f}}+\varrho_{\mathrm{f}}(1-\phi) \mathbf{b}-\mathbf{m} .
\end{aligned}
$$

Eqs. (1)-(5) are valid wherever the variables are smooth. At discontinuities, these balances are replaced by mass and linear momentum jump balances, which follow from these equations, see [15] for details. In particular, the volume average flow velocity of the mixture suffers no jump across the singular surface:

$\left(\mathbf{q}^{+}-\mathbf{q}^{-}\right) \cdot \mathbf{n}_{\mathbf{I}}=0$. 
Here, $\mathbf{q}^{+}$and $\mathbf{q}^{-}$denote the approximate limits of $\mathbf{q}$ on both sides of a singular surface with normal $\mathbf{n}_{\mathbf{I}}$.

\subsection{Constitutive assumptions}

Eqs. (1)-(5) have been formulated without any specific assumption on the material properties of the solid and liquid component, which enter into the model equations by constitutive assumptions.

\subsubsection{Constitutive variables}

The first step in the derivation of a mathematical model from the general balance equations is the choice of appropriate constitutive variables, which enter into the terms describing specific material behaviour, in this case $\mathbf{T}_{\mathrm{s}}, \mathbf{T}_{\mathrm{f}}$ and $\mathbf{m}$. General principles guiding the choice of the set of constitutive variables were formulated by Truesdell and Noll [22], see also [19] for a recent review, and include the requirement of frame indifference or objectivity of the resulting equations [23], the principle of equipresence [22] demanding that all constitutive variables occur in all constitutive equations, and the more restrictive hypothesis of phase separation $[20,23,24]$ postulating that variables arising from bulk-phase exact quantities (in this case, $\mathbf{T}_{\mathrm{s}}$, and $\mathbf{T}_{\mathrm{f}}$ ) are functions only of variables from that phase. The application of these principles, combined with the assumption that the constitutive equations for $\mathbf{T}_{\mathrm{s}}$, and $\mathbf{T}_{\mathrm{f}}$ are given in the most general isotropic forms $[6,25,26]$ which are linear in the velocity fields, and that the equation for $\mathbf{m}$ is given in the most general isotropic form linear in the velocities, implies that constitutive equations of the types

$\mathbf{T}_{\mathrm{s}}=-p_{s} \mathbf{I}+\mu_{\mathrm{s}}\left[\nabla \mathbf{v}_{\mathrm{s}}+\left(\nabla \mathbf{v}_{\mathrm{s}}\right)^{\mathrm{T}}\right]+\lambda_{\mathrm{s}}\left(\nabla \cdot \mathbf{v}_{\mathrm{s}}\right) \mathbf{I}$,

$\mathbf{T}_{\mathrm{f}}=-p_{\mathrm{f}} \mathbf{I}+\mu_{\mathrm{f}}\left[\nabla \mathbf{v}_{\mathrm{f}}+\left(\nabla \mathbf{v}_{\mathrm{f}}\right)^{\mathrm{T}}\right]+\lambda_{\mathrm{f}}\left(\nabla \cdot \mathbf{v}_{\mathrm{f}}\right) \mathbf{I}$,

$\mathbf{m}=-\alpha \mathbf{v}_{\mathrm{r}}+\beta \nabla \phi+\gamma\left(\frac{\partial \mathbf{v}_{\mathrm{r}}}{\partial t}+\left(\mathbf{v}_{\mathrm{r}} \cdot \nabla\right) \mathbf{v}_{\mathrm{r}}\right)$

should be sought, see $[6,15]$ for details. The scalar functions $p_{\mathrm{s}}, \mu_{\mathrm{s}}$, and $\lambda_{\mathrm{s}}$ are functions of $\phi$ and $\varrho_{\mathrm{s}}$; and $p_{\mathrm{f}}, \mu_{\mathrm{f}}$ and $\lambda_{\mathrm{f}}$ are functions of $\phi$ and $\varrho_{\mathrm{s}}$. However, as $\varrho_{\mathrm{s}}$, and $\varrho_{\mathrm{f}}$ are constants, we simply write $p_{\mathrm{s}}=p_{\mathrm{s}}(\phi), \mu_{\mathrm{s}}=\mu_{\mathrm{s}}(\phi), \lambda_{\mathrm{s}}=$ $\lambda_{\mathrm{s}}(\phi), p_{\mathrm{f}}=p_{\mathrm{f}}(\phi), \mu_{\mathrm{f}}=\mu_{\mathrm{f}}(\phi)$ and $\lambda_{\mathrm{f}}=\lambda_{\mathrm{f}}(\phi)$. Similarly, we have $\alpha=\alpha(\phi), \beta=\beta(\phi)$ and $\gamma=\gamma(\phi)$. Eq. (8) shows that both the solid and the fluid are modelled as viscous linear fluids.

Of course, more general treatments including additional effects are possible with a larger set of constitutive variables and assumptions that are more general than that of linear dependence. For example, additional interfacial forces such as Faxén or Basset forces could then be included in Eq. (9) (see [19], Chapter 18 for details).

\subsubsection{Solid and liquid component viscous stress tensors}

The quantities $p_{\mathrm{s}}$ and $p_{\mathrm{f}}$ in Eq. (8) are the solid- and liquid-component phase pressures. The viscous stress tensors $\mathbf{T}_{\mathrm{s}}{ }^{\mathrm{E}}$ and $\mathbf{T}_{\mathrm{f}}{ }^{\mathrm{E}}$ are defined through
$\mathbf{T}_{s}=-p_{\mathrm{s}} \mathbf{I}+\mathrm{T}_{\mathrm{s}}^{\mathrm{E}}, \quad \mathbf{T}_{\mathrm{f}}=-p_{\mathrm{f}} \mathbf{I}+\mathbf{T}_{\mathrm{f}}^{\mathrm{E}}$.

The functions $\mu_{\mathrm{s}}(\phi)$ and $\mu_{\mathrm{f}}(\phi)$ are the phase shear viscosities. For simplicity, and since this assumption is frequently made [27], it is assumed that solid and fluid phase bulk viscosities or expansion viscosities, defined by $\kappa_{\mathrm{s}}(\phi)=$ $\lambda_{\mathrm{s}}(\phi)+2 / 3 \mu_{\mathrm{s}}(\phi)$ and $\kappa_{\mathrm{f}}(\phi)=\lambda_{\mathrm{f}}(\phi)+2 / 3 \mu_{\mathrm{f}}(\phi)$, respectively, vanish. A complete treatment of the general case is presented in [15].

The constitutive equations for the viscous stress tensors can now be rewritten in the standard form of a viscous linear fluid behaviour [27]:

$\mathbf{T}_{\mathrm{s}}^{\mathrm{E}}=\mu_{\mathrm{s}}(\phi)\left[\nabla \mathbf{v}_{\mathrm{s}}+\left(\nabla \mathbf{v}_{\mathrm{s}}\right)^{\mathrm{T}}-\frac{2}{3}\left(\nabla \cdot \mathbf{v}_{\mathrm{s}}\right) \mathbf{I}\right]$,

$\mathbf{T}_{\mathrm{f}}^{\mathrm{E}}=\mu_{\mathrm{f}}(\phi)\left[\nabla \mathbf{v}_{\mathrm{f}}+\left(\nabla \mathbf{v}_{\mathrm{f}}\right)^{\mathrm{T}}-\frac{2}{3}\left(\nabla \cdot \mathbf{v}_{\mathrm{f}}\right) \mathbf{I}\right]$.

The phase shear viscosities $\mu_{\mathrm{s}}(\phi)$ and $\mu_{\mathrm{f}}(\phi)$ are not to be confused with the dynamical shear viscosities of the pure solid and of the pure liquid, respectively (see [27]).

\subsubsection{Partial pressures, pore pressure and effective solid stress}

The solid and liquid partial pressures $p_{\mathrm{f}}$ and $p_{\mathrm{s}}$ are theoretical variables, which cannot be measured experimentally. They are replaced by the experimental variables pore pressure $\tilde{p}$ and effective solid stress $\sigma_{\mathrm{e}}$, which satisfy

$p:=p_{\mathrm{s}}+p_{\mathrm{f}}=\tilde{p}+\sigma_{\mathrm{e}}$

where $p$ denotes the total pressure. Drawing from experience of soil mechanics [28], $\sigma_{\mathrm{e}}$ is considered to be a non-negative function of $\phi$. The concept of effective solid stress was developed for cases in which the solid particles have permanent contact with each other. Nevertheless, during hindered settling solid stresses can develop due to particle-particle collisions. This collision solid stress is assumed to be constant, but the solid stress enters the balance equations through its gradient only. Let $\phi_{c}$ be a critical concentration at which the solid particles begin to touch each other. Since the effective solid stress should be a non-decreasing function of the particle concentration, we assume that a constitutive equation for $\sigma_{\mathrm{e}}=\sigma_{\mathrm{e}}(\phi)$ is given satisfying

$\sigma^{\prime}{ }_{\mathrm{e}}(\phi):=\frac{\mathrm{d} \sigma_{\mathrm{e}}}{\mathrm{d} \phi} \begin{cases}=0 & \text { for } \phi \leq \phi_{\mathrm{c}} \\ >0 & \text { for } \phi>\phi_{\mathrm{c}}\end{cases}$

Some authors assume that $\sigma_{\mathrm{e}}^{\prime}$ is discontinuous at $\phi=\phi_{\mathrm{c}}$ [7,29], while others [28] have suggested that the effective solid stress is zero for hindered settling and that its transition to full value in consolidation occurs within a range of concentrations rather than at a determined value, such that $\sigma_{\mathrm{e}}^{\prime}$ could be given by a continuous function.

While the pore pressure $\tilde{p}$ is defined within the fluid filling the interstices of the solid, the partial fluid pressure is defined in the fluid component occupying the whole volume of the mixture. To express $p_{\mathrm{s}}$ and $p_{\mathrm{f}}$ in terms of $\tilde{p}$ and $\sigma_{\mathrm{e}}$, consider a settling column of cross section $\mathcal{S}$. Let $\mathcal{S}_{\mathrm{f}} \subset \mathcal{S}$ be the part of 
the cross section filled out by the fluid in the porous medium and define the surface porosity $\varepsilon:=\left|\mathcal{S}_{\mathrm{f}}\right| /|\mathcal{S}|$, i.e. $\mathrm{d} \mathcal{S}_{\mathrm{f}}=\varepsilon \mathrm{d} \mathcal{S}$. Then the surface forces exerted on the fluid in a cross section of the sediment are

$\int_{\mathcal{S}} p_{\mathrm{f}} \mathrm{d} \mathcal{S}=\int_{\mathcal{S}_{\mathrm{f}}} \tilde{p} \mathrm{~d} \mathcal{S}_{\mathrm{f}}=\int_{\mathcal{S}} \tilde{p}(\varepsilon \mathrm{d} \mathcal{S})$.

In general, the surface porosity should be given as a function of the volume porosity, i.e. of the volumetric solid concentration, $\varepsilon=\varepsilon(\phi)$. For example, if the solid flocs consist of cohesive spheres, $\varepsilon=1-\phi^{2 / 3}$ is appropriate. However, if no particular geometry of the solid flocs is taken into account, we may assume that all variables are horizontally constant in the settling column and that $\phi$ varies continuously beneath the surface of the sediment. This implies that the surface porosity equals the volume porosity, $\varepsilon(\phi)=1-\phi$. Using the localisation theorem [21], it is then easy to deduce from Eqs. (10)-(12) that

$p_{\mathrm{f}}=(1-\phi) \tilde{p}$,

$p_{s}=\phi \tilde{p}+\sigma_{\mathrm{e}}$,

respectively (see [18]). In terms of $\tilde{p}$ and $\sigma_{\mathrm{e}}$, the linear momentum balances of the components (Eqs. (4) and (5)), can be written as:

$$
\begin{aligned}
\varrho_{\mathrm{s}} \phi\left(\frac{\partial \mathbf{v}_{\mathrm{s}}}{\partial t}+\left(\mathbf{v}_{\mathrm{s}} \cdot \nabla\right) \mathbf{v}_{\mathrm{s}}\right)= & -\nabla(\phi \tilde{p})-\nabla \sigma_{\mathrm{e}}+\nabla \cdot \mathbf{T}_{\mathrm{s}}^{\mathrm{E}} \\
& -\varrho_{\mathrm{s}} \phi g \mathbf{k}+\mathbf{m}, \\
\varrho_{\mathrm{f}}(1-\phi)\left(\frac{\partial \mathbf{v}_{\mathrm{f}}}{\partial t}+\left(\mathbf{v}_{\mathrm{f}} \cdot \nabla\right) \mathbf{v}_{\mathrm{f}}\right) & =-\nabla((1-\phi) \tilde{p})+\nabla \cdot \mathbf{T}_{\mathrm{f}}^{\mathrm{E}} \\
& -\varrho_{\mathrm{f}}(1-\phi) g \mathbf{k}-\mathbf{m} .
\end{aligned}
$$

Furthermore, the fluid flow in a porous bed of solids, such as the sediment in a thickener, depends on the pore pressure in excess of the hydrostatic pressure $\varrho_{\mathrm{f}} g(H-z)$, where $H$ is the height of the vessel under consideration, rather than on its absolute value. Therefore, the excess pore pressure

$p_{\mathrm{e}}:=\tilde{p}-\varrho_{\mathrm{f}} g(H-z)$

is a more appropriate variable. However, this variable is introduced only in the final form of the model equations.

\subsubsection{Solid-fluid interaction force}

The general constitutive Eq. (9) for the solid-fluid interaction force $\mathbf{m}$ corresponds to a decomposition of this variable into a hydrostatic part $\mathbf{m}_{\mathrm{b}}$ and a dynamic part $\mathbf{m}_{\mathrm{d}}$, i.e. $\mathbf{m}=\mathbf{m}_{\mathrm{b}}+\mathbf{m}_{\mathrm{d}}$, where $\mathbf{m}_{\mathrm{b}}:=\beta \nabla \phi$ and

$\mathbf{m}_{\mathrm{d}}:=-\alpha(\phi) \mathbf{v}_{\mathrm{r}}+\gamma(\phi)\left(\frac{\partial \mathbf{v}_{\mathrm{r}}}{\partial t}+\left(\mathbf{v}_{\mathrm{r}} \cdot \nabla\right) \mathbf{v}_{\mathrm{r}}\right)$.

Note that $\mathbf{m}_{\mathrm{d}}$ vanishes for $\mathbf{v}_{\mathrm{r}} \equiv 0$. Therefore, the function $\beta$ can be determined by considering Eq. (16) for $\mathbf{v}_{\mathrm{s}}=\mathbf{v}_{\mathrm{f}}$. Assuming that $\mathbf{v}_{\mathrm{s}} \equiv 0, \mathbf{v}_{\mathrm{f}} \equiv 0$ and $\nabla \tilde{p}=-\varrho_{\mathrm{f}} g \mathbf{k}$ corresponding to the equilibrium state attained for $t \rightarrow \infty$ in a settling column $[18,23,30]$ yields

$\mathbf{m}_{\mathrm{b}}=\beta \nabla \phi=\tilde{p} \nabla \phi$.

The function $\alpha(\phi)$ is the resistance coefficient and assumed to be given by a constitutive equation. The virtual mass $\gamma(\phi)$ will not be determined since it turns out in Section 2.5 that the advective acceleration term in Eq. (18) can be neglected.

\subsubsection{Phase- and mixture-representative viscosities}

We define the mixture-representative shear viscosity $\mu_{\text {mix }}(\phi)=\mu_{\mathrm{s}}(\phi)+\mu_{\mathrm{f}}(\phi)$ and the function $h(\phi)=\mu_{\mathrm{s}}(\phi) / \mu_{\text {mix }}$ $(\phi)$. The viscous stress tensor of the mixture is then defined as

$\mathbf{T}^{\mathrm{E}}:=\mathbf{T}_{\mathrm{I}}^{\mathrm{E}}-\frac{\varrho_{\mathrm{s}} \varrho_{\mathrm{f}} \phi(1-\phi)}{\varrho(\phi)} \mathbf{v}_{\mathrm{r}} \mathbf{v}_{\mathrm{r}}$,

where

$\mathbf{T}_{\mathrm{I}}^{\mathrm{E}}:=\mathbf{T}_{\mathrm{s}}^{\mathrm{E}}+\mathbf{T}_{\mathrm{f}}^{\mathrm{E}}$

is called the inner part of the viscous stress tensor $\mathbf{T}^{\mathrm{E}}$ [14]. The second term in Eq. (20) comes from the diffusion of linear momentum due to solid-fluid relative motion. Defining a mixture-representative velocity gradient by using $h(\phi)$ as a weighting function between $\nabla \mathbf{v}_{\mathrm{s}}$, and $\nabla \mathbf{v}_{\mathrm{f}}$, i.e.

$\nabla \mathbf{v}_{\text {mix }}:=h(\phi) \nabla \mathbf{v}_{\mathrm{s}}+(1-h(\phi)) \nabla \mathbf{v}_{\mathrm{f}}$,

the inner part of $\mathbf{T}^{\mathrm{E}}$ takes the same form as the viscous stress tensor for a single-phase viscous linear fluid,

$\mathbf{T}_{\mathrm{I}}^{\mathrm{E}}=\mu_{\text {mix }}(\phi)\left[\nabla \mathbf{v}_{\text {mix }}+\left(\nabla \mathbf{v}_{\text {mix }}\right)^{\mathrm{T}}-\frac{2}{3}\left(\nabla \cdot \mathbf{v}_{\text {mix }}\right) \mathrm{I}\right]$.

Obviously, any two of the functions $\mu_{\mathrm{s}}(\phi), \mu_{\mathrm{f}}(\phi), \mu_{\text {mix }}(\phi)$ and $h(\phi)$ can be prescribed independently. The physical interpretation of $\mu_{\text {mix }}(\phi)$ and appropriate choices of the viscosity functions are given in Section 2.7.

\subsection{Solid-fluid relative velocity and linear momentum balance of the mixture}

The assumptions introduced in Section 2.3 are now used to obtain an equation for the solid-fluid relative velocity $\mathbf{v}_{\mathbf{r}}$ and the linear momentum balance of the mixture from the solid and fluid component momentum balance.

\subsubsection{Solid-fluid relative velocity}

Inserting Eq. (19) into Eqs. (15) and (16), we obtain, respectively,

$$
\begin{aligned}
\nabla \sigma_{\mathrm{e}}= & -\varrho_{\mathrm{s}} \phi\left(\frac{\partial \mathbf{v}_{\mathrm{s}}}{\partial t}+\left(\mathbf{v}_{\mathrm{s}} \cdot \nabla\right) \mathbf{v}_{\mathrm{s}}\right)-\phi \nabla \tilde{p}+\nabla \cdot \mathbf{T}_{\mathrm{s}}^{\mathrm{E}} \\
& -\varrho_{\mathrm{s}} \phi g \mathbf{k}-\alpha(\phi) \mathbf{v}_{\mathrm{r}}+\gamma(\phi)\left(\frac{\partial \mathbf{v}_{\mathrm{r}}}{\partial t}+\left(\mathbf{v}_{\mathrm{r}} \cdot \nabla\right) \mathbf{v}_{\mathrm{r}}\right)
\end{aligned}
$$




$$
\begin{aligned}
\nabla \tilde{p}= & -\varrho_{\mathrm{f}}\left(\frac{\partial \mathbf{v}_{\mathrm{f}}}{\partial t}+\left(\mathbf{v}_{\mathrm{f}} \cdot \nabla\right) \mathbf{v}_{\mathrm{f}}\right)-\varrho_{\mathrm{f}} g \mathbf{k}+\frac{1}{1-\phi} \\
& \times\left[\nabla \cdot \mathbf{T}_{\mathrm{f}}^{\mathrm{E}}+\alpha(\phi) \mathbf{v}_{\mathrm{r}}-\gamma(\phi)\left(\frac{\partial \mathbf{v}_{\mathrm{r}}}{\partial t}+\left(\mathbf{v}_{\mathrm{r}} \cdot \nabla\right) \mathbf{v}_{\mathrm{r}}\right)\right] .
\end{aligned}
$$

For reasons of notational convenience to become evident later, the resistance coefficient is replaced by the Kynch batch flux density function $f_{\mathrm{bk}}$ defined by

$f_{\mathrm{bk}}(\phi):=-\frac{\Delta \varrho g \phi^{2}(1-\phi)^{2}}{\alpha(\phi)}$.

To obtain an expression for $\mathbf{v}_{\mathrm{r}}$, we insert Eq. (25) into Eq. (24) to obtain

$$
\begin{aligned}
\mathbf{v}_{\mathrm{r}}= & \frac{f_{\mathrm{bk}}(\phi)}{\Delta \varrho g \phi^{2}(1-\phi)}\left[\nabla \sigma_{\mathrm{e}}(\phi)+\phi \Delta \varrho g \mathbf{k}\right. \\
& +\frac{\phi}{1-\phi} \nabla \cdot \mathbf{T}_{\mathrm{f}}^{\mathrm{E}}-\nabla \cdot \mathbf{T}_{\mathrm{s}}^{\mathrm{E}} \\
& +\phi\left(\varrho_{\mathrm{s}}\left(\frac{\partial \mathbf{v}_{\mathrm{s}}}{\partial t}+\left(\mathbf{v}_{\mathrm{s}} \cdot \nabla\right) \mathbf{v}_{\mathrm{s}}\right)\right. \\
& \left.-\varrho_{\mathrm{f}}\left(\frac{\partial \mathbf{v}_{\mathrm{f}}}{\partial t}+\left(\mathbf{v}_{\mathrm{f}} \cdot \nabla\right) \mathbf{v}_{\mathrm{f}}\right)\right) \\
& \left.-\frac{1}{1-\phi} \gamma(\phi)\left(\frac{\partial \mathbf{v}_{\mathrm{r}}}{\partial t}+\left(\mathbf{v}_{\mathrm{r}} \cdot \nabla\right) \mathbf{v}_{\mathrm{r}}\right)\right]
\end{aligned}
$$

The dimensional analysis in Section 2.5 will justify reducing Eq. (27) to

$\mathbf{v}_{\mathrm{r}}=\frac{f_{\mathrm{bk}}(\phi)}{\Delta \varrho g \phi^{2}(1-\phi)}\left[\nabla \sigma_{\mathrm{e}}(\phi)+\phi \Delta \varrho g \mathbf{k}\right]$

i.e. since $f_{\mathrm{bk}}$ and $\sigma_{\mathrm{e}}$, are given functions, the relative velocity $\mathbf{v}_{\mathrm{r}}$ is a known function of $\phi$ and $\nabla \phi$. The derivation of Eq. (27) and eventually of Eq. (28) from the solid and fluid linear momentum balances is analogous to the derivation of Darcy's law in the theory of mixtures (see [20]).

\subsubsection{Linear momentum balance of the mixture}

Adding Eqs. (4) and (5) yields the linear momentum balance of the mixture, which is written here as

$$
\begin{aligned}
\varrho(\phi) & \left(\frac{\partial \mathbf{v}}{\partial t}+(\mathbf{v} \cdot \nabla) \mathbf{v}\right)+\nabla \cdot\left(\frac{\varrho_{\mathrm{s}} \varrho_{\mathrm{f}} \phi(1-\phi)}{\varrho(\phi)} \mathbf{v}_{\mathrm{r}} \mathbf{v}_{\mathrm{r}}\right) \\
= & -\varrho(\phi) g \mathbf{k}-\nabla p+\mu_{\text {mix }}(\phi) \nabla^{2} \mathbf{q}+\left(\nabla \mu_{\text {mix }}(\phi)\right) \\
& \times\left[\nabla \mathbf{q}+(\nabla \mathbf{q})^{\mathrm{T}}\right]-\nabla \cdot\left(\mu _ { \text { mix } } ( \phi ) \left[( \phi - h ( \phi ) ) \left[\nabla \mathbf{v}_{\mathrm{r}}\right.\right.\right. \\
& \left.+\left(\nabla \mathbf{v}_{\mathrm{r}}\right)^{\mathrm{T}}-\frac{2}{3}\left(\nabla \cdot \mathbf{v}_{\mathrm{r}}\right) \mathbf{I}\right]+\nabla \phi \mathbf{v}_{\mathrm{r}}+\left(\nabla \phi \mathbf{v}_{\mathrm{r}}\right)^{\mathrm{T}} \\
& \left.\left.-\frac{2}{3}\left(\nabla \phi \cdot \mathbf{v}_{\mathrm{r}}\right) \mathbf{I}\right]\right),
\end{aligned}
$$

where $\varrho(\phi)=\varrho_{\mathrm{s}} \phi+\varrho_{\mathrm{f}}(1-\phi)$ is the local density of the mixture and

$\mathbf{v}=\frac{1}{\varrho(\phi)}\left(\varrho_{\mathrm{s}} \phi \mathbf{v}_{\mathrm{s}}+\varrho_{\mathrm{f}}(1-\phi) \mathbf{v}_{\mathrm{f}}\right)$ its local mass average velocity. In view of the condition $\nabla \cdot \mathbf{q}=0$, Eq. (29) is written in this particular way in order to obtain a perturbation of the Navier-Stokes equations for incompressible flows to determine $\mathbf{q}$ and $p$. Bürger et al. [15] show, using the kinematic relationships

$\phi \mathbf{v}_{\mathrm{s}}=\phi(1-\phi) \mathbf{v}_{\mathrm{r}}+\phi \mathbf{q}, \quad \mathbf{v}=\mathbf{q}+r(\phi) \mathbf{v}_{\mathrm{r}}$,

where $r(\phi):=\Delta \varrho \phi(1-\phi) / \varrho(\phi)$, that the mass balances of the solid component and of the mixture, Eqs. (1) and (3), and Eq. (29) which is equivalent to one of the two momentum balances (Eqs. (4) and (5)), can be rewritten in terms of the velocities $\mathbf{q}$ and $\mathbf{v}_{\mathbf{r}}$ as

$$
\begin{aligned}
& \frac{\partial \phi}{\partial t}+\nabla \cdot\left(\phi(1-\phi) \mathbf{v}_{\mathrm{r}}+\phi \mathbf{q}\right)=0 \\
& \nabla \cdot \mathbf{q}=0 \\
& -\varrho(\phi)\left[\frac{\partial \mathbf{q}}{\partial t}+(\mathbf{q} \cdot \nabla) \mathbf{q}+\frac{\partial\left(r(\phi) \mathbf{v}_{\mathrm{r}}\right)}{\partial t}\right. \\
& \quad+\left(\left(r(\phi) \mathbf{v}_{\mathrm{r}}\right) \cdot \nabla\right)\left(r(\phi) \mathbf{v}_{\mathrm{r}}\right)+(\mathbf{q} \cdot \nabla)\left(r(\phi) \mathbf{v}_{\mathrm{r}}\right) \\
& \left.\quad+\left(\left(r(\phi) \mathbf{v}_{\mathrm{r}}\right) \cdot \nabla\right) \mathbf{q}\right]-\nabla \cdot\left(\frac{\varrho_{\mathrm{s}} \varrho_{\mathrm{f}} \phi(1-\phi)}{\varrho(\phi)} \mathbf{v}_{\mathrm{r}} \mathbf{v}_{\mathrm{r}}\right) \\
& \quad+\mu_{\operatorname{mix}}(\phi) \nabla^{2} \mathbf{q}-\nabla p \\
& =\varrho(\phi) g \mathbf{k}-\left(\nabla \mu_{\operatorname{mix}}(\phi)\right) \cdot\left[\nabla \mathbf{q}+(\nabla \mathbf{q})^{\mathrm{T}}\right]+\nabla \\
& \quad \times\left(\mu _ { \operatorname { m i x } } ( \phi ) \left[(\phi-h(\phi))\left[\nabla \mathbf{v}_{\mathrm{r}}+\left(\nabla \mathbf{v}_{\mathrm{r}}\right)^{\mathrm{T}}-\frac{2}{3}\left(\nabla \cdot \mathbf{v}_{\mathrm{r}}\right) \mathrm{I}\right]\right.\right. \\
& \left.\left.\quad+\nabla \phi \mathbf{v}_{\mathrm{r}}+\left(\nabla \phi \mathbf{v}_{\mathrm{r}}\right)^{\mathrm{T}}-\frac{2}{3}\left(\nabla \phi \cdot \mathbf{v}_{\mathrm{r}}\right) \mathbf{I}\right]\right)
\end{aligned}
$$

The relationship (Eq. (27)) for $\mathbf{v}_{\mathrm{r}}$, which is equivalent to a second of the momentum balances (Eqs. (15) and (16)), completes these equations. The right-hand part of Eq. (27) can also be written out in terms of $\mathbf{v}_{\mathbf{r}}$ and $\mathbf{q}$, yielding a long expression (Eq. 37 in [15]) which, for sake of brevity, is not repeated here.

\subsection{Dimensional analysis}

A dimensional analysis is performed now in order to estimate the order of magnitude of the different terms in Eqs. (30)-(32) and of the expression (Eq. (27)) for $\mathbf{v}_{\mathbf{r}}$, which has not been written out here explicitly.

As in [18,31], the depth $L$ of the sedimentation vessel is chosen as a characteristic length, and we define a dimensionless gradient of a dimensionless flow quantity $u^{*}$ by $\nabla^{*} u^{*}=L \nabla u^{*}$. We assume $L \gg d$, where $d$ is a characteristic size of a single floc. For a rigorous treatment, there should exist two different typical velocities, the global motion of the mixture given by the velocity field $\mathbf{q}$ corresponds to a typical velocity $U$, while the local solid-fluid relative motion defined by $\mathbf{v}_{\mathrm{r}}$ is typified by a possibly different velocity $u_{\infty}$ (see [27]). Appropriate dimensionless velocities are then $\mathbf{q} *=U^{-1} \mathbf{q}$ and $\mathbf{v}_{\mathrm{r}}^{*}=U^{-1} \mathbf{v}_{\mathrm{r}}$. However, in the application considered, the flow is buoyancy-driven and we 
might take $U=u_{\infty}$, and a unique characteristic time can then be chosen by $t_{0}=L / u_{\infty}$. This assumption may not be valid for sedimentation-consolidation processes considered, for example, in rivers. The densities and the viscosities are non-dimensionalised by the mass density of the fluid, $\varrho_{\mathrm{f}}$, and by the dynamic shear viscosity of the fluid, $\mu_{0}$, respectively. The remaining dimensionless variables are then

$$
\begin{gathered}
p^{*}=\frac{p}{\varrho_{\mathrm{f}} g L}, \quad \sigma_{\mathrm{e}}^{*}(\phi)=\frac{\sigma_{\mathrm{e}}(\phi)}{\varrho_{\mathrm{f}} g L}, \\
f_{\mathrm{bk}}^{*}(\phi)=\frac{f_{\mathrm{bk}}(\phi)}{u_{\infty}}, \quad \gamma^{*}(\phi)=\frac{\gamma(\phi)}{\varrho_{\mathrm{f}}} .
\end{gathered}
$$

Two frequently employed parameters are then the Froude number of the flow and the sedimentation Reynolds number given by the respective equations

$\mathrm{Fr}:=\frac{\mathrm{U}^{2}}{g L}=\frac{u_{\infty}^{2}}{g L}, \quad \operatorname{Re}_{\mathrm{sed}}:=\frac{d u_{\infty}}{v_{0}}$,

where $\nu_{0}=\mu_{0} / \varrho_{f}$ denotes a typical kinematic viscosity. In dimensionless variables, Eqs. (30) and (31) read the same as before with all variables starred and are not repeated here, while the equation for $\mathbf{v}_{\mathrm{r}}$ takes the form

$$
\begin{aligned}
\mathbf{v}_{\mathrm{r}}^{*}= & \frac{f_{\mathrm{bk}}^{*}(\phi)}{\Delta \varrho^{*} \phi^{2}(1-\phi)}\left(\left[\nabla^{*}\left(\sigma_{\mathrm{e}}^{*}(\phi)\right)+\phi \Delta \varrho^{*} \mathbf{k}\right]\right. \\
& \left.+\frac{d}{L} \frac{\operatorname{Fr}}{\operatorname{Re}_{\mathrm{sed}}}\{\ldots\}_{1}+\operatorname{Fr}\{\ldots\}_{2}\right)
\end{aligned}
$$

where $\{\ldots\}_{1}$ contains viscous stress and $\{\ldots\}_{2}$ advective acceleration and virtual mass terms. We assume that all dimensionless variables and their derivatives are of order of magnitude $\mathcal{O}(1)$, so that the dimensionless parameters can be considered as estimates on the possible order of magnitude of the terms (in dimensional form) they multiply. In a similar way, Eq. (32) can be rewritten in dimensionless variables as

$$
\begin{aligned}
& \operatorname{Fr}\{\ldots\}_{3}+\frac{d}{L} \frac{\operatorname{Fr}}{\operatorname{Re}_{\text {sed }}} \mu_{\text {mix }}^{*}(\phi)\left(\nabla^{*}\right)^{2} \mathbf{q}^{*}-\nabla^{*} p^{*} \\
& \quad=\varrho^{*}(\phi) \mathbf{k}-\frac{d}{L} \frac{\operatorname{Fr}}{\operatorname{Re}_{\text {sed }}}\{\ldots\}_{4} .
\end{aligned}
$$

Here, $\{\ldots\}_{3}$ represents the dimensionless version of the advective acceleration and diffusion stress terms appearing as the two first terms on the left-hand part of Eq. (32), while $\{\ldots\}_{4}$ denotes the dimensionless form of the viscous stress terms appearing as the two last terms on the right-hand part of that equation. It should be pointed out that $\mathbf{v} *$ and its dimensionless derivatives occur linearly in the expressions $\{\ldots\}_{3}$ and $\{\ldots\}_{4}$. Both Eqs. (34) and (35) are written out completely in [15].

For the sedimentation-consolidation processes considered here, we assume that the parameters $u_{\infty}=10^{-4} \mathrm{~m} / \mathrm{s}$ (falling velocity of a single floc in an unbounded medium), $d=$ $10^{-4} \mathrm{~m}$ (assumed size of a single floc), $L=1 \mathrm{~m}$ (height of the sedimentation vessel), $v_{0}=10^{-6} \mathrm{~m}^{2} / \mathrm{s}$ (kinematic viscosity of water) and $g=10 \mathrm{~m} / \mathrm{s}^{2}$ are representative. Thus, we obtain

$\mathrm{Fr}=10^{-9}, \quad \operatorname{Re}_{\mathrm{sed}}=10^{-2}, \quad \frac{d}{L} \frac{\mathrm{Fr}}{\mathrm{Re}_{\mathrm{sed}}}=10^{-11}$.

Maintaining the terms $\{\ldots\}_{1}$ and $\{\ldots\}_{2}$ in Eq. (34) and inserting this definition of $\mathbf{v}_{\mathrm{r}} *$ into Eq. (35) will introduce $\mathcal{O}\left(\mathrm{Fr}^{2} d \operatorname{Re}_{\text {sed }}^{-2} L^{-1}\right)$ and $\mathcal{O}\left(\mathrm{Fr}^{2} d \mathrm{Re}_{\text {sed }}^{-1} L^{-1}\right)$ terms into the expressions $\{\ldots\}_{3}$ and $\{\ldots\}_{4}$ of Eq. (35), which are very small can be neglected. Therefore, we discard the terms $\{\ldots\}_{1}$ and $\{\ldots\}_{2}$ in Eq. (34), and obtain an explicit representation of the solid-fluid relative velocity as a function of $\phi$ and $\nabla \phi$ :

$\mathbf{v}_{\mathrm{r}}=\frac{f_{\mathrm{bk}}(\phi)}{\Delta \varrho g \phi^{2}(1-\phi)}\left[\nabla \sigma_{\mathrm{e}}(\phi)+\Delta \varrho g \phi \mathbf{k}\right]$.

There is no such obvious way to simplify Eq. (35) without further assumptions. If we decide to neglect both the advective acceleration terms and the viscous stress terms which are multiplied by $\mathrm{Fr}$ and by $d \mathrm{FrRe}_{\mathrm{sed}}{ }^{-1} L^{-1}$, respectively, we obtain the equation $\nabla p=\varrho(\phi) g \mathbf{k}$ or, replacing the total pressure $p$ by the effective solid stress $\sigma_{\mathrm{e}}$, and the excess pore pressure $p_{\mathrm{e}}$,

$\nabla p_{\mathrm{e}}=-\nabla\left(\sigma_{\mathrm{e}}(\phi)\right)-\Delta \varrho g \phi \mathbf{k}$,

in which the volume average flow velocity $\mathbf{q}$ no longer occurs. This quantity could then no longer be determined from the model equations; see Section 2.7.

\subsection{Final form of the model equations}

With the diffusion coefficient

$a(\phi):=-\frac{f_{\mathrm{bk}}(\phi) \sigma_{\mathrm{e}}^{\prime}(\phi)}{\Delta \varrho g \phi}$,

the continuity equations are

$\frac{\partial \phi}{\partial t}+\nabla \cdot\left(\phi \mathbf{q}+f_{\mathrm{bk}}(\phi) \mathbf{k}\right)=\nabla \cdot(a(\phi) \nabla \phi)$,

$\nabla \cdot \mathbf{q}=0$.

Although no terms are deleted from Eq. (35), this equation is rewritten in dimensional variables in a different form to make analogy with the Navier-Stokes equations apparent:

$$
\begin{aligned}
- & \varrho(\phi)\left(\frac{\partial \mathbf{q}}{\partial t}+(\mathbf{q} \cdot \nabla) \mathbf{q}\right)+\mu_{\operatorname{mix}}(\phi) \nabla^{2} \mathbf{q}-\nabla p_{\mathrm{e}} \\
= & \nabla \sigma_{\mathrm{e}}(\phi)+\Delta \varrho g \phi \mathbf{k}-\nabla\left(\mu_{\operatorname{mix}}(\phi)\right) \cdot\left[\nabla \mathbf{q}+(\nabla \mathbf{q})^{\mathrm{T}}\right] \\
& +\varrho(\phi)\left((\mathbf{q} \cdot \nabla)\left(r(\phi) \mathbf{v}_{\mathrm{r}}\right)+\left(\left(r(\phi) \mathbf{v}_{\mathrm{r}}\right) \cdot \nabla\right) \mathbf{q}\right) \\
& +\boldsymbol{\Psi}_{1}\left(\mathrm{D}_{x}^{\alpha_{1}} \phi\right)+\boldsymbol{\Psi}_{2}\left(\mathrm{D}_{x}^{\alpha_{2}} \phi, \frac{\partial \phi}{\partial t}\right),
\end{aligned}
$$

where $\boldsymbol{\Psi}_{1}$, and $\boldsymbol{\Psi}_{2}$ are non-linear known functions of $\phi$, its partial derivatives with respect to the space variable of maximal orders $\left|\alpha_{1}\right|$ and $\left|\alpha_{2}\right|$, respectively, and, in the case of 
$\Psi_{2}$, its derivative with respect to $t$. The explicit expressions for these functions are

$$
\begin{aligned}
\boldsymbol{\Psi}_{1}:= & \nabla \cdot\left(\mu _ { \operatorname { m i x } } ( \phi ) \left[( \phi - h ( \phi ) ) \left(\nabla \mathbf{v}_{\mathrm{r}}+\left(\nabla \mathbf{v}_{\mathrm{r}}\right)^{\mathrm{T}}\right.\right.\right. \\
& \left.-\frac{2}{3}\left(\nabla \cdot \mathbf{v}_{\mathrm{r}}\right) \mathbf{I}\right)+\nabla \phi \mathbf{v}_{\mathrm{r}}+\left(\nabla \phi \mathbf{v}_{\mathrm{r}}\right)^{\mathrm{T}} \\
& \left.\left.-\frac{2}{3}\left(\nabla \phi \cdot \mathbf{v}_{\mathrm{r}}\right) \mathbf{I}\right]\right) \\
\boldsymbol{\Psi}_{2}:= & \varrho(\phi) \Delta \varrho\left[\frac{\partial}{\partial t}\left(\frac{\phi(1-\phi)}{\varrho(\phi)} \mathbf{v}_{\mathrm{r}}\right)\right. \\
& \left.+\frac{\phi(1-\phi)}{\varrho(\phi)}\left(\mathbf{v}_{\mathrm{r}} \cdot \nabla\right)\left(\frac{\phi(1-\phi)}{\varrho(\phi)} \mathbf{v}_{\mathrm{r}}\right)\right] \\
& +\varrho_{\mathrm{s}} \varrho_{\mathrm{f}} \nabla \cdot\left(\frac{\phi(1-\phi)}{\varrho(\phi)} \mathbf{v}_{\mathrm{r}} \mathbf{v}_{\mathrm{r}}\right),
\end{aligned}
$$

in which the relative velocity $\mathbf{v}_{\mathrm{r}}$ is obtained from Eq. (36) as a known function of $\phi$ and $\nabla \phi$. Physically, the terms $\boldsymbol{\Psi}_{1}$ and $\boldsymbol{\Psi}_{2}$ model the interaction of the concentration field $\phi$ with the average flow field $\mathbf{q}$ and the excess pore pressure distribution $p_{\mathrm{e}}$. This interaction is produced by viscous stress due to concentration inhomogeneities and by advective acceleration due to solid-liquid relative motion and diffusion stress, respectively. For a homogeneous suspension, when $\phi$ is constant, $\mathbf{v}_{\mathrm{r}}$, is a constant vector and $\boldsymbol{\Psi}_{1}$ and $\boldsymbol{\Psi}_{2}$ vanish. For $\phi \equiv 0$, Eqs. (39) and (40) recover the Navier-Stokes system. Note that the multiindices $\alpha_{1}$, and $\alpha_{2}$ satisfy $\left|\alpha_{1}\right| \leq$ 3 and $\left|\alpha_{2}\right| \leq 2$, respectively. In particular, $\boldsymbol{\Psi}_{1}$ will in general depend on derivatives of $\phi$ up to third order.

\subsection{Discussion of the model equations}

\subsubsection{Type degeneracy of Eq. (38)}

Assume that the function $f_{\mathrm{bk}}$ satisfies Kynch's assumptions [1] $f_{\mathrm{bk}}(0)=f_{\mathrm{bk}}\left(\phi_{\max }\right)=0$ and $f_{\mathrm{bk}}(\phi)<0$ for $0<$ $\phi<\phi_{\max }$, where $\phi_{\max }$ is a maximum concentration. Then we obtain

$a(\phi) \begin{cases}=0 & \text { for } \phi \leq \phi_{\mathrm{c}} \text { and } \phi=\phi_{\max }: \text { Eq. (38) is } \\ & \text { hyperbolic, } \\ >0 & \text { for } \phi_{\mathrm{c}}<\phi<\phi_{\max }: \text { Eq. }\end{cases}$

i.e. Eq. (38) is of strongly degenerating parabolic or hyperbolic-parabolic type. Solutions of such equations are in general discontinuous. Some implications of this unusual behaviour will be demonstrated explicitly in the one-dimensional set-up.

\subsubsection{Choice of the viscosity functions}

There are two obvious choices of $h(\phi)$ and the resulting significance of $\mu_{\text {mix }}(\phi)$,

2.7.2.1. Model 1. Note that $\left|\alpha_{1}\right| \leq 2$ only if $h(\phi)=\phi$ for all $\phi$. Since $\phi$ is in general discontinuous, the function $\Psi_{1}$ should involve as few derivatives of $\phi$ as possible, therefore, we set $h(\phi)=h_{1}(\phi):=\phi$. Then we have

$\mu_{\mathrm{s}}(\phi)=\phi \mu_{\mathrm{mix}}(\phi), \quad \mu_{\mathrm{f}}(\phi)=(1-\phi) \mu_{\text {mix }}(\phi)$.
To make Model 1 complete, one of the viscosities $\mu_{\mathrm{s}}(\phi)$, $\mu_{\mathrm{f}}(\phi)$ or $\mu_{\text {mix }}(\phi)$ must be given.

2.7.2.2. Model 2. Consider the mixture as a single-phase viscous-linear flowing fluid. Then we may assume that the mixture viscosity $\mu_{\text {mix }}(\phi)$ coincides with its effective viscosity $\mu_{\text {eff }}(\phi)$, which is experimentally measurable, in contrast to what is at present known for the quantities $\left(\mu_{\mathrm{f}}(\phi)\right)$ or $\mu_{\mathrm{s}}(\phi)$. Moreover, we may assume that for all values of $\phi$ the viscosity $\mu_{\mathrm{eff}}(\phi)$ is that of a homogeneous mixture of globally constant concentration $\phi$. This assumption implies $\mathbf{v}_{\mathrm{r}}=$ constant, hence the diffusion stress terms vanish, i.e. the viscous stress tensor of the mixture $\mathbf{T}^{\mathrm{E}}$ coincides with its inner part $\mathbf{T}_{\mathrm{I}}{ }^{\mathrm{E}}$. If the mixture is assumed to behave as a single-phase viscous linear fluid, then $\mathbf{T}^{\mathrm{E}}$ should be expressed in terms of the same velocity as that occurring in the acceleration terms, i.e. in terms of $\mathbf{v}$. In view of Eqs. (22) and (23), this is achieved for $h(\phi)=h_{2}(\phi):=\varrho_{\mathrm{s}} \phi / \varrho(\phi)$. Then we will have

$$
\begin{aligned}
\mu_{\mathrm{s}}(\phi) & =\frac{\varrho_{\mathrm{s}} \phi}{\varrho(\phi)} \mu_{\mathrm{eff}}(\phi), \quad \mu_{\mathrm{f}}(\phi)=\left(1-\frac{\varrho_{\mathrm{s}} \phi}{\varrho(\phi)}\right) \mu_{\mathrm{eff}}(\phi) \\
& =\frac{\varrho_{\mathrm{f}}(1-\phi)}{\varrho(\phi)} \mu_{\mathrm{eff}}(\phi)
\end{aligned}
$$

Of course, by this choice, the term $\Psi_{1}$ involves third-order derivatives of $\phi$ in Eq. (40).

Both choices seem to present advantages and disadvantages, and a compromise between Model 1 and Model 2 could be to use $h_{1}(\phi)$ and to replace $\mu_{\text {mix }}(\phi)$ by $\mu_{\text {eff }}(\phi)$, for which semi-empirical laws such as [32]

$\mu_{\mathrm{eff}}(\phi)=\mu_{0}\left(1-\frac{\phi}{\phi_{\max }}\right)^{-2.5 \phi_{\max }}$,

are available, where $\mu_{0}$ denotes the viscosity of the pure fluid.

\subsubsection{The inviscid case}

If we assume that all viscous terms can be neglected, the model equations reduce to

$$
\begin{aligned}
& \frac{\partial \phi}{\partial t}+\nabla \cdot\left(\phi \mathbf{q}+f_{\mathrm{bk}}(\phi) \mathbf{k}\right)=\nabla \cdot(a(\phi) \nabla \phi), \\
& \nabla \cdot \mathbf{q}=0 \\
& \nabla p_{\mathrm{e}}=-\nabla \sigma_{\mathrm{e}}(\phi)-\Delta \varrho g \phi \mathbf{k} .
\end{aligned}
$$

A similar set of equations has been studied by Schneider $[33,34]$ for sedimentation of ideal suspensions, where the concept of effective solid stress is not included such that $a$ vanishes and Eq. (45) is of hyperbolic type. One of Schneider's conclusions is, however, also valid for the Eqs. (45)-(47): taking the curl of Eq. (47) yields that $\phi$ is a function only of the vertical co-ordinate $z$ and of $t$. Note that the Eqs. (45)-(47) are in general not sufficient to determine the average flow field $\mathbf{q}$, although under specific assumptions on the geometry, $\mathbf{q}$ can in some cases be obtained from suitable boundary conditions. 


\section{Results of mathematical analysis for the one-dimensional case}

In one space dimension, Eqs. (6) and (39) imply that $q$ depends only on $t$, and the model equations reduce to

$$
\begin{aligned}
\frac{\partial \phi}{\partial t}+ & \frac{\partial}{\partial z}\left(\phi q(t)+f_{\mathrm{bk}}(\phi)\right)=\frac{\partial}{\partial z}\left(a(\phi) \frac{\partial \phi}{\partial z}\right) \\
\frac{\partial p_{\mathrm{e}}}{\partial z}= & -\frac{\partial \sigma_{\mathrm{e}}(\phi)}{\partial z}-\Delta \varrho g \phi-\frac{\varrho(\phi) q(t)}{g} \\
& \times \frac{\partial}{\partial z}\left(\frac{f_{\mathrm{bk}}(\phi)}{\varrho(\phi) \phi}\left(\frac{\partial \sigma_{\mathrm{e}}(\phi)}{\partial z}+\Delta \varrho g \phi\right)\right)+\boldsymbol{\Psi}_{1}+\boldsymbol{\Psi}_{2}
\end{aligned}
$$

where $\boldsymbol{\Psi}_{1}$ and $\boldsymbol{\Psi}_{2}$ are the one-dimensional versions of the quantities defined in Eqs. (41) and (42). However, a calculation performed in [15] supports the view that in one space dimension, the terms $\boldsymbol{\Psi}_{1}$, and $\boldsymbol{\Psi}_{2}$ remain small, and that therefore the neglection of viscous stress terms considered by many authors are justified (see [7,17]).

Note that only solving Eq. (48) requires computational effort, since the excess pore pressure distribution can be determined a posteriori from Eq. (49) once $\phi$ has been calculated on the computational domain.

The one-dimensional case corresponds classically to the concept of an ideal continuous thickener (ICT), see Fig. 2, i.e. to solving Eqs. (48) and (49) on a space-time cylinder $\bar{Q}_{T}:=[0, L] \times[0, T]$. We restrict the discussion here to this set-up, due to Shannon and Tory [35] (see also Petty [36] and Bustos et al. [37]), in which the end $z=L$ of the space interval is identified with the feed source level, the end $z=0$ with the discharge mechanism, and into which the feed and discharge mechanisms enter as boundary conditions. Meanwhile, however, several authors including Lev et al. [38], Barton et al. [39], Diehl [40,41] and Concha et al. [43] have proposed and in part analysed one-dimensional sedimentation models in which the feed is modelled by an additional singular source term in Eq. (48), combined with discontinuities (with respect to $z$ ) in the volume average velocity $q$. These thickener-clarifier or high-capacity thickener models allow a description also of the clarification zone, i.e.

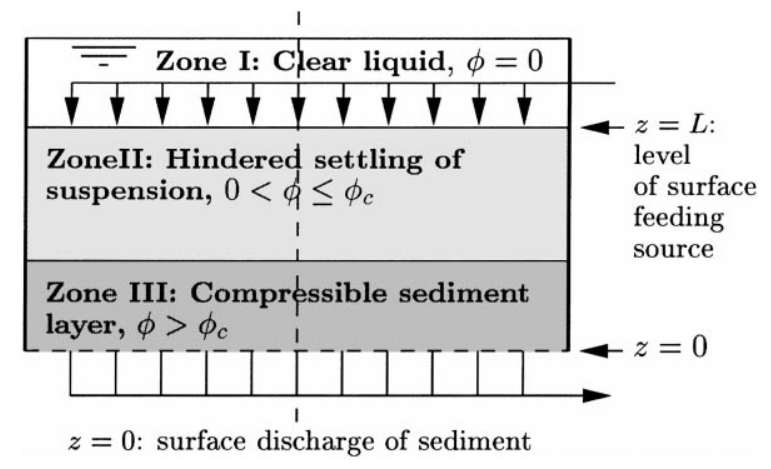

Fig. 2. Ideal continuous thickener (ICT). the part of the vessel above the feed level where normally clear liquid should overflow. Due to the singular source term, however, the mathematical treatment of these models is difficult and the theory has not yet risen to the same level of maturity as for the simpler ICT configuration characterised by Fig. 2. Some aspects of a thickener-clarifier model are also treated by Diehl's contribution to this issue [42].

Here, the value of $q(t)<0$ is prescribed by the total volumetric discharge of the mixture at $z=0, q(t)=-Q_{\mathrm{D}}(t) / \mathcal{U}$, where $Q_{D}(t)>0$ denotes the volumetric mixture discharge rate and $\mathcal{U}$ the assumed ICT unit cross-sectional area; consequently, at $z=0$, the solid volumetric flux density reduces to $q(t) \phi(0, t)$, from which we obtain the boundary condition at $z=0$,

$f_{\mathrm{bk}}(\phi)-\left.a(\phi) \frac{\partial \phi}{\partial z}\right|_{z=0}=0, \quad 0<t \leq T$.

A special case is an ICT without discharge operating as a settling column, which corresponds to $q \equiv 0$. Moreover, an initial concentration distribution

$\phi(z, 0)=\phi_{0}(z), \quad 0 \leq z \leq L$

and a boundary concentration value

$\phi(L, t)=\phi_{L}(t), \quad 0<t \leq T$

are prescribed. Defining the continuous Kynch flux density function

$f(\phi, t):=q(t) \phi+f_{\mathrm{bk}}(\phi)$

and assuming that $\phi_{L}(t)<\phi_{\mathrm{c}}$, condition Eq. (52) can be replaced by prescribing the feed flux,

$\left.f(\phi, t)\right|_{z=L}=f_{\mathrm{F}}(t), \quad 0<t \leq T$,

which is related to $\phi_{L}(t)$ by

$q(t) \phi_{L}(t)+f_{\mathrm{bk}}\left(\phi_{L}(t)\right)=f_{\mathrm{F}}(t), \quad 0<t \leq T$.

Eq. (48) and conditions (50)-(52) form an initial-boundary value problem. The salient difficulty encountered in the mathematical analysis is the type degeneracy, which is exacerbated by the mentioned frequent assumption that $\sigma_{\mathrm{e}}^{\prime}(\phi)$ (and thus $a(\phi)$ ) is discontinuous at $\phi=\phi_{\mathrm{c}}$. Common effective solid stress functions having this property include the approach

$\sigma_{\mathrm{e}}(\phi)=0$ for $\phi \leq \phi_{\mathrm{c}}$

$\sigma_{\mathrm{e}}(\phi)=k\left[\left(\frac{\phi}{\phi_{\mathrm{c}}}\right)^{n}-1\right]$ for $\phi \leq \phi_{\mathrm{c}}, k>0, n \geq 1$

(see [12] for references to the use of this expression). The non-linearity of the flux density function, which can, for example, be given by Michaels and Bolger's [44] semi-empirical approach

$$
\begin{aligned}
f_{\mathrm{bk}}(\phi) & =v_{\infty} \phi\left(1-\frac{\phi}{\phi_{\max }}\right)^{C}, \\
v_{\infty} & <0, \quad 0<\phi_{\max } \leq 1, \quad C \geq 1,
\end{aligned}
$$


and the degeneracy of Eq. (48) due to the vanishing of the diffusion coefficient $a(\phi)$ on an interval of solution values will cause solutions to be discontinuous in general. As has been pointed out elsewhere [45], these agents of discontinuities are independent from each other.

Discontinuous solutions have to be defined in a weak sense. Therefore Eq. (48) is multiplied by a sufficiently smooth test function, then integrated over the computational domain $\bar{Q}_{T}$, and then integration by parts and the boundary conditions are used to move derivatives from the sought solution to the test function. The concept of weak solutions is based on the requirement that the integral equation resulting from these transformations, in which the unknown solution is no longer differentiated, holds for all test functions from a suitably chosen set. However, weak solutions are not unique, and additional entropy conditions or selection criteria are necessary to select the physically relevant one, the entropy weak solution, from the family of weak solutions.

Assuming that $\sigma_{\mathrm{e}}^{\prime}(\phi)$ and hence $a(\phi)$ are smooth, and that the initial and boundary data of the problem, $\phi_{0}(z)$ and $\phi_{L}(t)$, satisfy smoothness and compatibility assumptions, Bürger and Wendland [46] proved existence and uniqueness of entropy weak solutions of the resulting initial-boundary value problem. The presentation of their definition would require mathematical preliminaries that are not within the scope of this paper and is not given here, see the limited outline in [10], Chapter 9 and their original paper [46] for details. However, it is instructive to state explicitly the particular jump condition Bürger and Wendland [47] have shown to be valid for their entropy weak solutions.

It is well known that discontinuities of entropy weak solutions of the hyperbolic equation

$\frac{\partial \phi}{\partial t}+\frac{\partial}{\partial z}\left(q(t) \phi+f_{\mathrm{bk}}(\phi)\right)=0$

between two solution values $\phi^{+}$and $\phi^{-}\left(\phi^{+}\right.$is the value above and $\phi^{-}$the value below the jump) propagate at the speed $\sigma=\sigma\left(\phi^{+}, \phi^{-}\right)$given by the Rankine-Hugoniot jump condition

$\sigma\left(\phi^{+}, \phi^{-}\right)=q(t)+\frac{f_{\mathrm{bk}}\left(\phi^{+}\right)-f_{\mathrm{bk}}\left(\phi^{-}\right)}{\phi^{+}-\phi^{-}}$,

and that this discontinuity is admissible only if Oleĭnik's jump entropy condition,

$$
\begin{aligned}
& \frac{f_{\mathrm{bk}}(u)-f_{\mathrm{bk}}\left(\phi^{-}\right)}{u-\phi^{-}} \geq \sigma\left(\phi^{+}, \phi^{-}\right)-q(t) \\
& \geq \frac{f_{\mathrm{bk}}(u)-f_{\mathrm{bk}}\left(\phi^{+}\right)}{u-\phi^{+}} \text {for all } u \text { between } \phi^{-} \text {and } \phi^{+},
\end{aligned}
$$

is satisfied, in which case the discontinuity is called a shock.

A typical discontinuity in the context of sedimentation is the interface between the sediment formed on the bottom of an ICT and the supernatant liquid. The fact that the propagation velocity of this interface is merely determined by $\phi^{-}$ and $\phi^{+}$, and the easy geometrical interpretation of Eq. (54) using the graph of $f_{\mathrm{bk}}(\phi)$ or of $f(\phi, t)$ (in most circumstances, $q$ is assumed to be constant), have made it possible to construct explicit entropy weak solutions in Kynch's sedimentation theory by the method of characteristics, see $[37,48]$. Most notably, a control model for continuous sedimentation in an ICT has been based on the a priori knowledge of the sediment level growth or fall rate deduced from the Rankine-Hugoniot condition (see [49]).

Unfortunately, the situation is different in the phenomenological theory. Bürger and Wendland's jump condition derived in [47] implies that the propagation velocity of the sediment-suspension interface, separating the compression zone $\left(\phi>\phi_{c}\right)$ from the hindered settling zone $\left(\phi \leq \phi_{c}\right)$, involves an additional term in relation to the right-hand part of Eq. (53). At a point $(z, t)$ belonging to that interface,

$$
\begin{aligned}
\sigma(z, t)= & q(t)+\frac{1}{\phi^{+}-\phi_{\mathrm{c}}} \\
& \times\left[f_{\mathrm{bk}}\left(\phi^{+}\right)-f_{\mathrm{bk}}\left(\phi_{\mathrm{c}}\right)+\lim _{\xi \rightarrow z} \frac{\partial}{\partial z} A(\phi)\right]
\end{aligned}
$$

holds, were $\phi^{+}$is the concentration of the suspension above the sediment and

$A(\phi)=\int_{0}^{\phi} a(s) d s$

The jump entropy condition (Eq. (54)) has to be modified in a similar way. Eq. (55) shows that also the limit of the derivative of $\phi$ with respect to $z$, taken from below the sediment level, enters into the propagation speed. However, this limit is in general unknown a priori, and the construction of a control model of continuous sedimentation, based on the full phenomenological model in which $\sigma_{\mathrm{e}}^{\prime}(\phi)$ is not assumed to vanish, is still an open problem.

Careful analysis is also required for the treatment of the boundary conditions: solution values propagating along straight-lined characteristics might intersect the boundary of the computational domain at $z=L$ from the interior. Such a situation corresponds to the overflow of the ICT. The solution value $\phi_{L}(t)$ can then no longer prescribed in a pointwise sense, and the boundary condition (Eq. (52)) has to be replaced by a more general set-valued boundary condition, a so-called entropy boundary condition, in order to ensure well-posedness of the initial-boundary value problem, see $[47,50]$ for details.

The analysis of the one-dimensional initial-boundary value problem (Eqs. (48) and (50)-(52)) has been continued by Bürger et al. in [45], where the same problem is treated under considerably relaxed regularity assumptions. In particular, existence and uniqueness of entropy weak solutions has been shown under the assumption that $\sigma_{\mathrm{e}}^{\prime}(\phi)$ is discontinuous, which had not been admitted in the previous analysis [46]. 


\section{Conclusions}

The last paragraph has shown that any non-trivial solution of Eqs. (48) and (50)-(52) requires numerical solution procedures, and the companion paper by Bürger et al. [11] provides guidelines for the design of appropriate numerical techniques and shows that it is possible to solve the phenomenological model numerically with reasonable effort. The case study by Bürger et al. [12] in this issue complements the mathematical analysis, showing that the model is not only mathematically sound but indeed applies to real suspensions. We refer to these papers for the conclusions that can be drawn for these two important aspects of the phenomenological theory outlined here and limit ourselves to the discussion of some aspects of the extension to multidimensions, which apparently requires the treatment of phenomena not encountered in one space dimension.

Such new effects seem to be related to the terms $\boldsymbol{\Psi}_{1}$, and $\boldsymbol{\Psi}_{2}$, which describe the coupling between the flow field and the kinematic waves given by the evolution of the concentration distribution. It had been pointed out by Schneider [34] that this phenomenon is intrinsically multidimensional, and a first step should be a quantitative analysis to determine the possible order of magnitude of these expressions.

It should be mentioned that results of the dimensional analysis have been used in a very conservative way, i.e. if a concrete flow problem is to be solved, the Froude and sedimentation Reynolds numbers may take values justifying the additional neglection either of the advective acceleration or of the viscous terms in the linear momentum balance. In other words, this balance still offers possibilities for simplifications not performed (and not advocated unless concrete materials are specified) here.

Another challenging open problem is that of determining appropriate phase and mixture viscosities and is related to finding a suitable formulation for the mixture stress, which has attracted the interest of many authors. For example, Rudman [51] suggests to start the modelling from the balance equation of the mixture formulated in terms of the volume average velocity $\mathbf{q}$ and using the effective viscosity $\mu_{\text {eff }}$ of the mixture, as in the compromise between Model 1 and Model 2, and to derive the component balances subsequently from the mixture balances.

Of course, the practical task of determining effective viscosity functions for given suspensions has received much more attention (see, e.g. Acrivos [52] and Happel and Brenner [53]) than the theoretical problem of splitting this quantity into phase-representative viscosities, although the solution of the latter seems fundamental for the complete understanding of the theory of mixtures and their application to sedimentation-consolidation processes. Ungarish [27] has recognised this: he proposes equations which are equivalent to using $\mu_{\text {eff }}(\phi)$ as the mixture viscosity and to setting $h(\phi)=$ constant, and at the same time strongly emphasises that the resulting splitting of $\mu_{\text {eff }}$ into phase viscosities is quite rough and due to lack of better informa- tion. Consequently, the postulation of phase-representative viscosities for formal reasons still requires physical and experimental justification.

Although the implications of the phenomenological model in several space dimensions are still far from being well understood, the steps of progress in the modelling of one-dimensional sedimentation-consolidation processes this theory has given rise to let its extension to multidimensions appear to be a rewarding task.

\section{Acknowledgements}

The author would like to thank the United Engineering Foundation and the Sonderforschungsbereich 404 at the University of Stuttgart for support, which made the preparation of this paper and its presentation at the conference possible.

\section{References}

[1] G.J. Kynch, Trans. Faraday Soc. 48 (1952) 166.

[2] K.J. Scott, Trans. Inst. Min. Met. 77 (1968) C85.

[3] F.M. Auzerais, R. Jackson, W.B. Russel, J. Fluid Mech. 195 (1988) 437.

[4] R. Buscall, L.R. White, J. Chem. Soc. Faraday Trans. 83 (1987) 873.

[5] O. Bascur, Modelo fenomenológico de suspensiones en sedimentación, Engineering thesis, Department of Metallurgical Engineering, University of Concepción, Chile, 1976.

[6] F. Concha, M.C. Bustos, in: B.M. Moudgil, P. Somasundaran (Eds.), Flocculation, Sedimentation and Consolidation, AIChE, New York, 1986, p. 275.

[7] K.A. Landman, L.R. White, Adv. Colloid Interf. Sci. 51 (1994) 175.

[8] M.C. Bustos, On the existence and determination of discontinuous solutions to hyperbolic conservation laws in the theory of sedimentation, Doctoral thesis, Faculty of Mathematics, TH Darmstadt, Germany, 1984.

[9] R. Bürger, Ein Anfangs-Randwertproblem einer quasilinearen parabolischen entarteten Gleichung in der Theorie der Sedimentation mit Kompression, Doctoral thesis, Faculty of Mathematics, University of Stuttgart, 1996.

[10] M.C. Bustos, F. Concha, R. Bürger, E.M. Tory, Sedimentation and Thickening, Kluwer Academic Publishers, Dordrecht, The Netherlands, 1999.

[11] R. Bürger, S. Evje, K.H. Karlsen, K.-A. Lie, Chem. Eng. J. 80 (2000) 91-104.

[12] R. Bürger, F. Concha, F.M. Tiller, Chem. Eng. J. 80 (2000) 105-117.

[13] F. Dobran, Int. J. Multiphase Flow 11 (1985) 1.

[14] C. Truesdell, Rational Thermodynamics, second ed., Springer, New York, 1984

[15] R. Bürger, W.L. Wendland, F. Concha, Z. Angew. Math. Mech. 80 (2000) 79.

[16] J.C. Slattery, AIChE J. 13 (1967) 1066.

[17] S. Whitaker, Transp. Porous Media 1 (1986) 3.

[18] F. Concha, M.C. Bustos, A. Barrientos, E.M. Tory (Eds.), Sedimentation of Small Particles in a Viscous Fluid. Computational Mechanics Publications, Southampton, UK, 1996.

[19] D.A. Drew, S.L. Passman, Theory of Multicomponent Fluids, Springer, New York, 1999.

[20] K.R. Rajagopal, L. Tao, Mechanics of Mixtures, World Scientific, Singapore, 1995.

[21] M. Gurtin, An Introduction to Continuum Mechanics, Academic Press, San Diego, CA, USA, 1981. 
[22] C. Truesdell, W. Noll, in: S. Flügge ()Ed.), Handbuch der Physik, vol. III/3, Springer, Berlin, 1965.

[23] D.A. Drew, L.A. Segel, Stud. Appl. Math. 50 (1971) 205.

[24] S.L. Passman, J.W. Nunziato, E.K. Walsh, in: C. Truesdell (Ed.), Rational Thermodynamics, second ed., Springer, New York, 1984, p. 286.

[25] A. Barrientos, F. Concha, Mecánica Racional Moderna. Volumen 1: Fundamentos Matemáticos, University of Concepción, Chile, 1994.

[26] C.C. Wang, Arch. Rat. Mech. Anal. 36 (1970) 166.

[27] M. Ungarish, Hydrodynamics of Suspensions, Springer, Berlin, 1993.

[28] V. Pane, R.L. Schiffman, Géotechnique 35 (1985) 69.

[29] M.D. Green, M. Eberl, K.A. Landman, AIChE J. 42 (1996) 2308.

[30] D.A. Drew, Ann. Rev. Fluid Mech. 15 (1983) 261.

[31] A. Acrivos, E. Herbolzheimer, J. Fluid Mech. 92 (1979) 435.

[32] C.R. Wildemuth, M.C. Williams, Rheol. Acta 23 (1984) 627.

[33] W. Schneider, J. Fluid Mech. 120 (1982) 323.

[34] W. Schneider, in: G.E.A. Meier, F. Obermeier (Eds.), Flow of Real Fluids, Lecture Notes in Physics, Springer, Berlin, 1985, p. 326.

[35] P.T. Shannon, E.M. Tory, Trans. SME 235 (1966) 375.

[36] C.A. Petty, Chem. Eng. Sci. 30 (1975) 1451.

[37] M.C. Bustos, F. Concha, W.L. Wendland, Math. Methods Appl. Sci. 13 (1990) 1

[38] O. Lev, E. Rubin, M. Sheintuch, AIChE J. 32 (1986) 1516.
[39] N.G. Barton, C.-H. Li, S.J. Spencer, J. Aust. Math. Soc. Ser. B 33 (1992) 269-289.

[40] S. Diehl, SIAM J. Appl. Math. 56 (1996) 388.

[41] S. Diehl, SIAM J. Appl. Math. 57 (1997) 991.

[42] S. Diehl, Chem. Eng. J. 80 (2000) 119-133.

[43] F. Concha, A. Barrientos, M.C. Bustos, in: Proceedings of the XIX International Mineral Processing Congress, San Francisco, USA, 1995, p. 75 (Chapter 14).

[44] A.S. Michaels, J.C. Bolger, Ind. Eng. Chem. 1 (1962) 24.

[45] R. Bürger, S. Evje, K.H. Karlsen, J. Math. Anal. Appl., in press.

[46] R. Bürger, W.L. Wendland, J. Math. Anal. Appl. 218 (1998) 207.

[47] R. Bürger, W.L. Wendland, Math. Methods Appl. Sci. 21 (1998) 865.

[48] M.C. Bustos, F. Concha, Math. Methods Appl. Sci. 10 (1988) 245.

[49] M.C. Bustos, F. Paiva, W.L. Wendland, Math. Methods Appl. Sci. 12 (1990) 533.

[50] M.C. Bustos, F. Paiva, W.L. Wendland, Math. Methods Appl. Sci. 19 (1996) 679.

[51] M. Rudman, J. Aust. Math. Soc. Ser. B 39 (1997) 149.

[52] A. Acrivos, in: E. Guazzelli, L. Oger, Mobile Particulate Systems, Kluwer Academic Publishers, Dordrecht, The Netherlands, 1995.

[53] J. Happel, H. Brenner, Low Reynolds Number Hydrodynamics, Prentice Hall, Englewood Cliffs, NJ, USA, 1965. Republished by Martinus Nijhoff Publishers, Dordrecht, The Netherlands, 1986. 